\title{
The tumor suppressor gene RhoBTB1 is a novel target of miR-31 in human colon cancer
}

\author{
RUI-SI XU, XIAO-DONG WU, SI-QI ZHANG, CHANG-FENG LI, LEI YANG, DAN-DAN LI, \\ BAO-GANG ZHANG, YING ZHANG, JING-PENG JIN and BIN ZHANG
}

Endoscopy Center, China-Japan Union Hospital, Jilin University, Changchun 130033, P.R. China

Received August 29, 2012; Accepted October 18, 2012

DOI: 10.3892/ijo.2012.1746

\begin{abstract}
RNAs are a class of endogenous non-coding RNA, which can regulate downstream target genes through binding to the 3'UTR of those genes. Numerous studies have indicated that abnormal expression of miRNAs is implicated in tumor development. Aberrant expression of miR-31 has been found in various cancers, including colorectal cancer. Here, we show that miR-31 is upregulated in human colon cancer tissues and cell lines, and that repression of miR-31 inhibited colon cancer cell proliferation and colony formation in soft agarose. To further elucidate the mechanism underlying the role of miR-31 in promoting colon cancer, we used online miRNA target prediction databases and found that the tumor suppressor RhoTBT1 may be a target of miR-31. Imunohistochemistry assay revealed that RhoBTB1 was significantly decreased in HT29 cells. In addition, ectopic expression of miR-31 reduced RhoBTB1 in the colon cancer cell line HT29. The results suggested that suppression of RhoBTB1 may be responsible for colon tumorigenesis, which was inhibited directly by miR-31. The results of MTT and soft agarose colony-formation assays showed that knockdown of RhoBTB1 by RNAi induced cell proliferation, and colony formation in soft agarose, which mimicked the function of miR-31. This further suggested that suppression of RhoBTB1 was responsible for colon tumorigenesis. In conclusion, we found that miR-31 acts as an oncogene in colon cancer and identified RhoBTB1 as a new target of miR-31 further study demonstrated that miR-31 contributed to the development of colon cancer at least partly by targeting RhoBTB1.
\end{abstract}

\section{Introduction}

Colon cancer is one of the most common cancers and is the third leading cause of cancer-related mortality worldwide (1).

Correspondence to: Dr Bin Zhang, Endoscopy Center, China-Japan Union Hospital, Jilin University, 126 Xiantai Street, Changchun 130033, P.R. China

E-mail: zhangbin_bio@163.com

Key words: RhoBTB1, miR-31, colon cancer, tumorigenesis
Up to now, the most efficient treatment of colon cancer is the radical surgery combined with chemotherapy, for local tumors, the 5-year survival rate is approximately $93 \%$ (2), while almost half of patients had developed metastasis on admission (3), even the localized disease, more than one-third of patients develop recurrence after surgery (4). Despite advances in surgical techniques and other treatments, even after the novel molecular agents in recent years, colon cancer high mortality rate remains because of the frequent recurrence and metastasis (5-7). Currently, the mechanisms underlying the preocess of colon tumorigenesis remain obscure, understanding the related mechanism is important for us to develop new stratege and find new efficient target for efficient treatment of colon cancer.

MicroRNAs (miRNAs) are a class of 19-24 nt short noncoding RNAs, which regulate translation and degraduation of target mRNAs by direct interactions with 3'-untranslated region (3'UTR) of the target mRNAs. Approximately 1,500 miRNA sequences have been found (8) and it was reported that $>60 \%$ of genes are conserved targets of microRNA in the human genome (9). It has been demonstrated that miRNAs can regulate the expression of a wide rage of genes, and play important roles in cell progression such as development (10), differentiation (11), proliferation $(12,13)$ and apoptosis $(14,15)$. Increasing evidence has suggested that miRNAs play a crucial role in carcinogenesis and tumor progression $(16,17)$, and many miRNAs were found upregulated or downregulated in various tumor types $(18,19)$.

MicroRNA-31 (miR-31) plays important roles in malignant tumors, dysregulation of it has been demonstrated to be involved in many tumors, for example, it was upregulated in head and neck cancer, hepatocellular carcinoma, lung cancer and colon cancer (20-24). Many reports have revealed that miR-31 was correlated to the ability of cancer metastasis. Inhibition of miR-31 contributed to induction of metastasis in breast cancer (25). Interestingly, Laurila et al found both inhibition and enhanced expression of miR-31 lead to reduced migration and invasion of pancreatic cancer cells (26). In colon cancer, miR-31 was reported to be significantly upregulated, and the expression level of which was correlated with the stage of colon cancer $(20,21)$ and miR-31 was also found to be associated with colon cancer invasion and metastasis, supression of miR-31 affected migration and invasion of colon cancer cells (27). It was also found that for colon cancer at early stage, supression of miR-31 could increase sensitivity to 5-FU (28). 
RhoBTB proteins belong to a subfamily of the Rho family of small GTPases. The RhoBTB subfamily comprises three members, RhoTBT1, RhoTBT2 and RhoTBT3. RhoTBT2 was first proposed as a tumor supressor, it has been reported that in breast cancer, lack of RHOBTB2 transcripts results in growth inhibition (29). Studies also found high rates of loss of heterozygosity at the RHOTBT2 locus in gastric tumors and bladder tumors $(30,31)$. Similarly to RhoTBT2, recently RhoTBT1 was also proposed as a tumor supressor in a study on head and neck cancer (32).

Athough miR-31 was demonstrated to be envolved in colon cancer, its mechanisms are still not clear. In this study, we found miR-31 was upregulated in colon cancer, and demonstrated for the first time that RhoBTB1 was a target of miR-31. We also explored the role of miR-31 in colon and revealed that miR-31 could promote colon tumorigenesis and invasiveness, at least partly through targeting RhoBTB1.

\section{Meterials and methods}

Tumor samples. Colon cacer tissues and mathed adjacent normal tissues were collected from 17 colon cancer patients. All carcinoma samples were obtained at the time of operation and obtained with the informed consent of the patients. All human materials were used in accordance with the policies of the institutional review board at China-Japan Union Hospital of Jilin University, China.

Cell culture and transfection. The HT29 and SW480 cell lines were from American Type Culture Collection (ATCC) and cultured under conditions provided by the manufacturer. Twentyfour hours before transfection cells were seeded, then miR-31 antisense locked nucleic acid (LNA anti-miR31) and control oligonucleotides (LAN control) or RhoBTB1 siRNA and control siRNA were transfected with Lipofectamine 2000 (Invitrogen, Carlsbad, CA) according to the manufacturer's instructions. Twenty-four or forty-eight hours later, cells were collected and subjected to further analysis. The human RhoBTB1 siRNA and control siRNA were purchased from Origene.

RNA extraction and real-time RT-PCR. Total RNA was extracted from cells and tumor tissues by using TRIzol (Invitrogen). For miR-31 detection, stem-loop RT-PCR assay was performed. U6 snRNA was used as an internal control. The following stem-loop primers were used for miRNA-31: 5'-GTCGTATC CAGTGCAGGGTCCGAGGTATTCGCACTGGATACGACA GCTAT-3' and U6 snRNA: 5'-GTCGTATCCAGTGCAGG GTCCGAGGTATTCGCACTGGATACGACAAAATATGG AAC-3'. For RhoBTB1 mRNA detection, M-MLV Reverse Transcriptase system (Promega) was used to reverse-transcribe RNA into cDNA. The qRT-PCR was carried out using the SYBR Green qPCR Master Mix (Tiangen) on ABI 7300 (Applied Biosystems, Foster, CA). Glyceraldehyde 3-phosphate dehydrogenase was used as an internal control. The primers used for detection of miR-31 and U6 were, forward: 5'-GGAGAGGCA AGATGCTGGCA-3'; U6-forward: 5'-CGCAAGGATGACAC GCAAATTC-3', and a universal downstream reverse primer, 5'-GTGCAGGGTCCGAGGT-3'. The primers used for detection of RhoBTB1, forward: 5'-GGAGTGAAGGAGCCTGTGAG-3'; reverse: 5'-TGCCAATGAACCCCTTACTC-3'. Internal control
GAPDH primers for qRT-PCR, forward: 5'-ACGGATTTGGT CGTATTGGG-3', reverse: 5'-TGATTTTGGAGGGATCT CGC-3'.

Luciferase reporter assays. Twenty-four hours before transfection HT29 cells were seeded into a 24 -well plate $\left(2.5-3 \times 10^{4} /\right.$ well), then the cells were cotransfected with Renilla luciferase and luciferase reporter plasmids containing miR-31 or vector control and wild-type or mutated target gene 3'UTR with Lipofectamine 2000 (Invitrogen). Forty-eight hours after transfection, luciferase activities were measured using dualluciferase reporter assay system (Promega). Firefly luciferase activities were normalized to Renilla luciferase.

Western blotting. After transfection as indicated, cells were harvested and lysed in RIPA lysis buffer [150 mM NaCl, $10 \mathrm{mM}$ Tris, $\mathrm{pH} 7.5,1 \%$ NP40, $1 \%$ deoxycholate, $0.1 \%$ SDS, protease inhibitor cocktail (Sigma)] and total protein was measured using the Bradford protein assay (Bio-Rad, Hercules, CA). Equal amounts of protein sample was subjected to $10 \%$ SDS-PAGE and transferred to PVDF membrane (Millipore, Billerica, MA, USA), after blocking in 5\% skimmed milk for $30 \mathrm{~min}$ at room temperature, the membrane was incubated in anti-RhoBTB1 (1:2000, Abcam) for $2 \mathrm{~h}$ at room temperature, followed by incubating with horseradish peroxidase-linked secondary antibody for $1 \mathrm{~h}$ at room temerature and visualized with ECL (Millipore). $\beta$-actin (1:5000, Abcam, Inc., Cambridge, MA) was used as loading control.

Immunohistochemistry (ICH) and in situ hybridization. Tumor or matched normal tissues were fixed with $4 \%$ paraformaldehyde, then the samples were embeded in paraffin and sectioned. Followed incubated with RhoBTB1 antibody (1:500), the bound antibodies were detected with the biotin-streptavidinperoxidase system (Vector Labs, Burlingame, CA, USA) using diaminobenzidine (Sigma-Aldrich) as chromogen.

For in situ hybridization assay, DIG-labeled locked nucleic acid (LNA)-based probe specific for miR-31 (Exiqon, Vedbaek, Denmark) was introduced. The in situ hybridization signal was detected by incubation with horseradish peroxidase (HRP)-conjugated anti-digoxigenin (1:200, Abcam Inc., Cambridge, MA).

MTT.Cell proliferation rates were measured using 3-(4,5-dimethylthiazol-2-yl)-2,5-diphenyltetrazolium bromide (MTT) reduction assay according to the manufacturer's instructios. In brief, HT29 cells were seeded into 96 -well plates $24 \mathrm{~h}$ before transfection and treated as described previously. Then MTT solution was added in each well, and the cells were cultured in incubator for another $4 \mathrm{~h}$. The medium was discarded and $100 \mu \mathrm{l}$ of DMSO was added to each cell, after shaking for 15 min the absorbance at $595 \mathrm{~nm}$ was measured with microplate reader (Bio-Rad).

Soft agar colony formation assay. Pre-warmed $1 \mathrm{ml}$ of 2X RPMI-1640 medium with 20\% fetal calf serum (FBS) and $1 \mathrm{ml}$ of $1.2 \%$ Bacto-agar (Difco) solution were mixed and transferred onto a $3-\mathrm{cm}$ dish, and then incubated at $4{ }^{\circ} \mathrm{C}$ for $30 \mathrm{~min}$ to allow the bottom agar layer to solidify. Next, mixed $0.5 \mathrm{ml}$ melted $0.6 \%$ agar at $50^{\circ} \mathrm{C}$ with equal volume of 

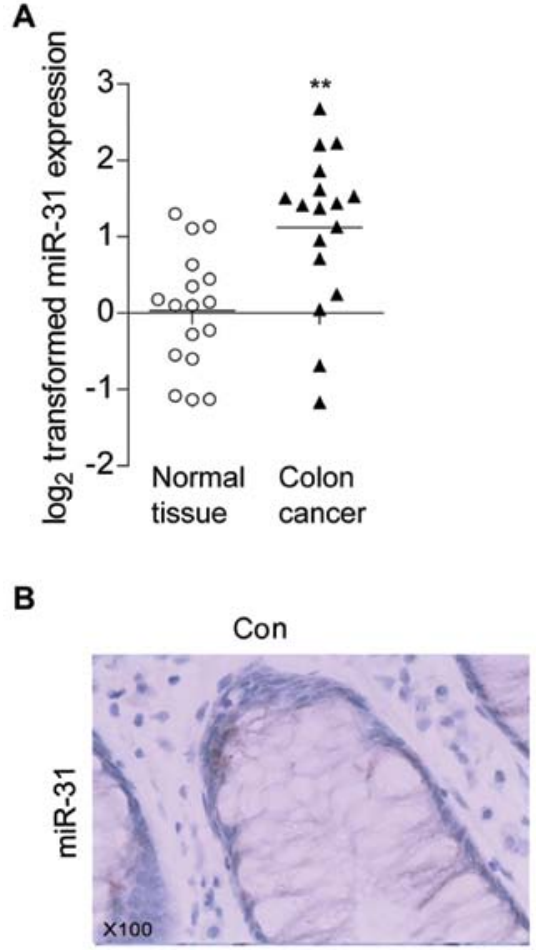

C
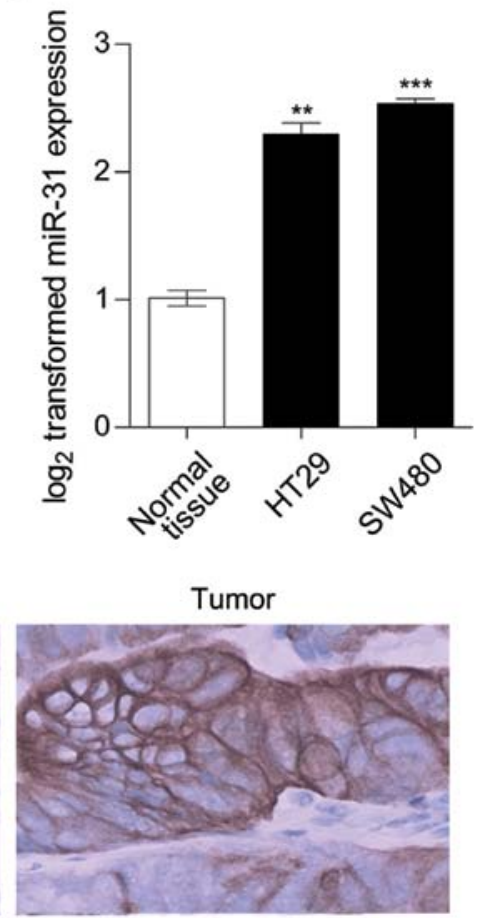

Figure 1. miR-31 expression is upregulated in human colon cancer tissues and cell lines. (A) Quantification of the overexpression of miR-31 by real-time RT-PCR assays in human colon cancers versus normal colon tissues. Error bars indicate SD. ${ }^{* *} \mathrm{P}<0.01$. (B) ISH assays for representative overexpressed miR-31 in colon cancers. These assays were conducted on human colon cancers and paired normal colon tissues. Overexpression of miR-31 was detected in malignant versus normal colon tissues. H\&E staining of the indicated tissue sections is shown. Original magnification x100. (C) miR-31 expression was examined by qRT-PCR in colon cancer cell lines as indicated. ${ }^{* *} \mathrm{P}<0.01 ;{ }^{* * * *} \mathrm{P}<0.01$.

$2 \mathrm{X}$ medium and stored in $40^{\circ} \mathrm{C}$ water bath, then $1 \times 10^{3}$ cells were added and mixed quickly and poured onto the bottom agar. Cells were left to grown for 2 weeks in the incubator, then cell colonies were observed under a microscope, and colonies of $>50$ cells were counted.

Statistical analysis. Data are presented as mean \pm SEM. Student's t-test (two-tailed) was used to compare two groups $(\mathrm{P}<0.05$ was considered significant) unless otherwise indicated $\left(\chi^{2}\right.$ test).

\section{Results}

miR-31 expression is upregulated in human colon cancer tissues and cell lines. It has been reported by many studies that miR-31 was dysregulated in various cancers, so we examined miR-31 expression level in human colon cancer tissues by using qRT-PCR. As showed in Fig. 1A, compared with the normal colon tissues miR-31 was significantly increased in tumor tissues. ISH assay results further confirmed that miR-31 was induced in colon cancer tissues (Fig. 1B). Moreover, we detected miR-31 expression levels in colon cancer cell lines HT29 and SW480. qRT-PCR results showed that compared with normal colon tissues, miR-31 was also increased in HT29 and SW480 (Fig. 1C), this was in accordance with the previous studies (19-21). These results suggested that miR-31 might be involved in colon tumor progression.
Repression of miR-31 affects human colon cancer cell viability and anchorage-independent colony formation. To examine the role of miR-31 in colon cancer development, we inhibited miR-31 in HT29 cells by using locked nucleic acid-modified anti-miR31 antisense (LNA anti-miR31). As showed in Fig. 2A (right), after tranfected with LNA anti-miR31, miR31 was effectively inhibited in HT29 cells. MTT assays showed that inhibition of miR-31 reduced viability of HT29 cells (Fig. 2A, left), which indicates that upregulation of miR-31 might attribute to colon tumorigenesis.

Anchorage-independence of cell growth abitity is a property of cancer cells. To verify the involvement of miR-31 in colon tumorigenesis we next performed soft agarose assays. The results showed, after knockdown of miR-31 colony formation in soft agarose was obviously supressed (Fig. 2B). Collectively the results demostrated that repression of miR-31 inhibited human colon cancer cell viability and anchorageindependent colony formation, which further confirmed that miR-31 plays an important role in colon cancer development.

miR-31 targets RhoBTB1. Since miRNAs regulate translation and degraduation of target mRNAs by direct interactions with 3'UTR of the target mRNAs, we investigated the targets of miR-31 to further uncover the mechanism underlying its tumor promoting effect on colon tumorigenesis. By using online miRNA target prediction databases (http://www.targetscan.org), we hypothesized that RhoBTB1 is a target of miR-31 (Fig. 3A). To investigate whether RhoBTB1 was directly targeted by 
A
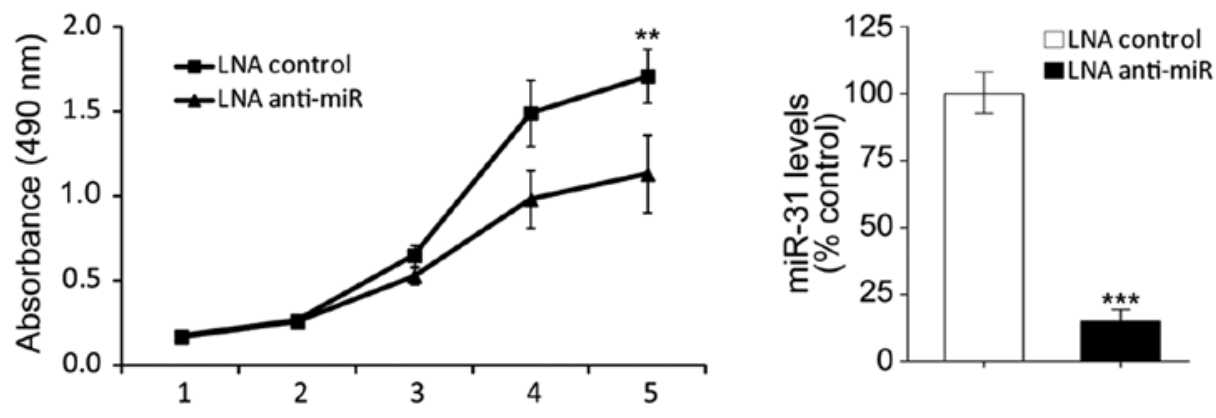

B
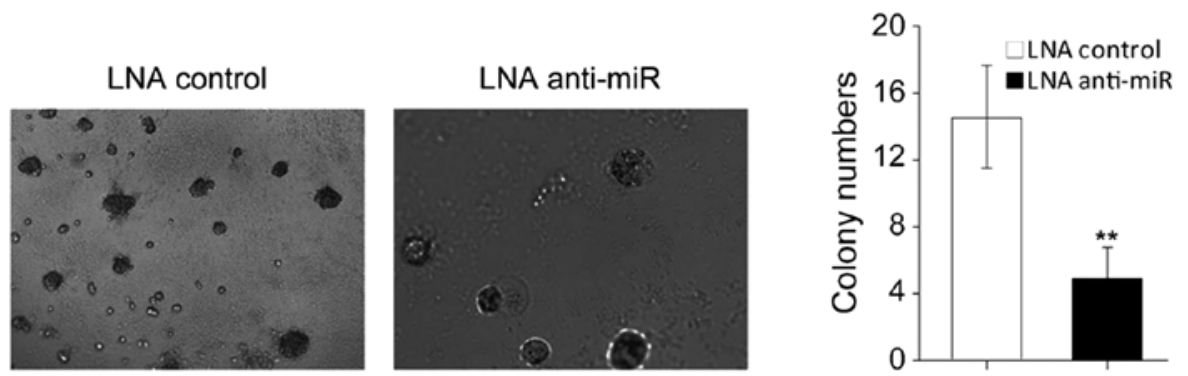

Figure 2. Repression of miR-31 expression affects human colon cancer cell viability and anchorage-independent colony formation. (A) Treatment with LAN anti-miR-31 LNA decreased proliferation. miR-31 expression was decreased with LNA anti-miR-31 treatment for $24 \mathrm{~h}$. HT29 cells were transfected with LAN control RNA or LAN anti-miR-31 as indicated. Cell viability was detected at the indicated time-points post-transfection using MTT assays. The right panel displays results of real-time assays that confirmed miR-31 repression. (B) Repression of miR-31 expression significantly affects human colon cancer cell clonal growth. Colony formation was suppressed in HT29 cells relative to controls by engineered knockdown of miR-31 through transient LAN anti-miR-31 transfection.

A

miR-31 target sites in $3^{\prime}$ UTR of RhoBTB1

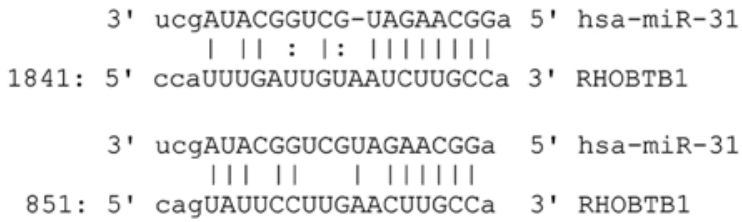

B
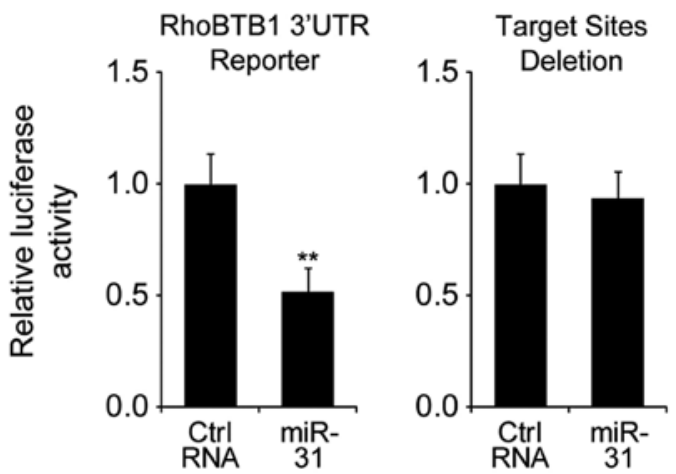

Figure 3. RhoBTB1 is a direct target of miR-31. (A) Tumor suppressor gene RhoBTB1 might be the potential target of miR-31. Sequence alignments of miR-31 and its target sites in 3'UTR of RhoBTB1, downloaded from http:// www.microrna.org are shown. (B) HT29 cells were co-transfected with human RhoBTB1 3'UTR firefly luciferase reporter plasmid or miR-31 target sites deleted reporter construct, together with RL plasmids, control RNA or miR-31 mimics as indicated. After $48 \mathrm{~h}$, firefly luciferase activity was measured and normalized by Renilla luciferase activity.

miR-31, we performed luciferase reporter gene assay in HT29 cells.
Firstly, we constructed luciferase reporter plasmids containing RhoBTB1 3'UTR sequence (wt-RhoBTB1) or with deleted putative miR-31 target sites (mut-RhoBTB1). As shown in Fig. 3B, after co-tranfected with wt-RhoBTB1 plasmid, the luciferase activity of miR-31 transfected cells was significantly reduced compared to control miR tranfected cells, while co-tranfected with mut-RhoBTB1 plasmid did not change the luciferase activity. Taken together, these results indicated that miR-31 directly regulated RhoBTB1 by targeting its 3'UTR.

miR-31 downregulates RhoBTB1 expression in HT29 cells. RhoBTB family belongs to the Rho family of small GTPases, which comprises three members, of which RhoBTB1 and RhoBTB2 have been proposed as tumor suppressors. Here we found that RhoBTB1 was a target of miR-31 in the colon cancer cell line HT29, then we investigated the effect of miR-31 on RhoBTB1. Compared with HT29 transfected with control RNA, RhoBTB1 mRNA level was significantly downregulated in miR-31 mimic-tansfected HT29 cells (Fig. 4A), and western blotting results showed RhoBTB1 protein expression level was also downregulated with miR-31 overexpression (Fig. 4B). These observations suggested that miR-31 upregulation in colon tumor might depress RhoBTB1.

RhoBTB1 expression is downregulated in human colon cancer tissues. As mentioned above, RhoTBT1 was proposed as a tumor supressor (32), and we also found that upregulation of miR-31 in colon cancer cell line HT29 could depressed RhoBTB1, then we performed ICH and western blotting experiments to examine the expression level of RhoBTB1 in colon cancer tissues. As showed in Fig. 5, RhoBTB1 was 


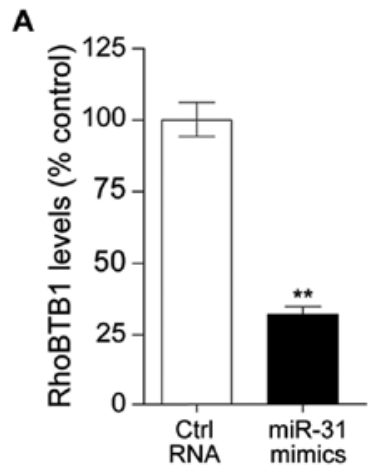

B

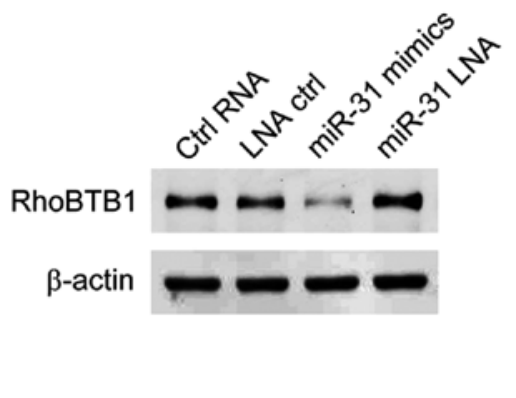

Figure 4. miR-31 downregulates RhoBTB1 expression in HT29 cells. (A) HT29 cells were transfected with control RNA or miR-31 mimics. After 48 h, RT-PCR assays confirmed that RhoBTB1 mRNA level was downregulated by pre-miR-31 transfections performed in HT29 cells. *** $\mathrm{P}<0.05$. (B) HT29 cells were transfected with control RNA or miR-31 mimics. After 48 h, RhoBTB1 and internal control $\beta$-actin were detected by western blotting. Similar results were obtained from three independent experiments.

A
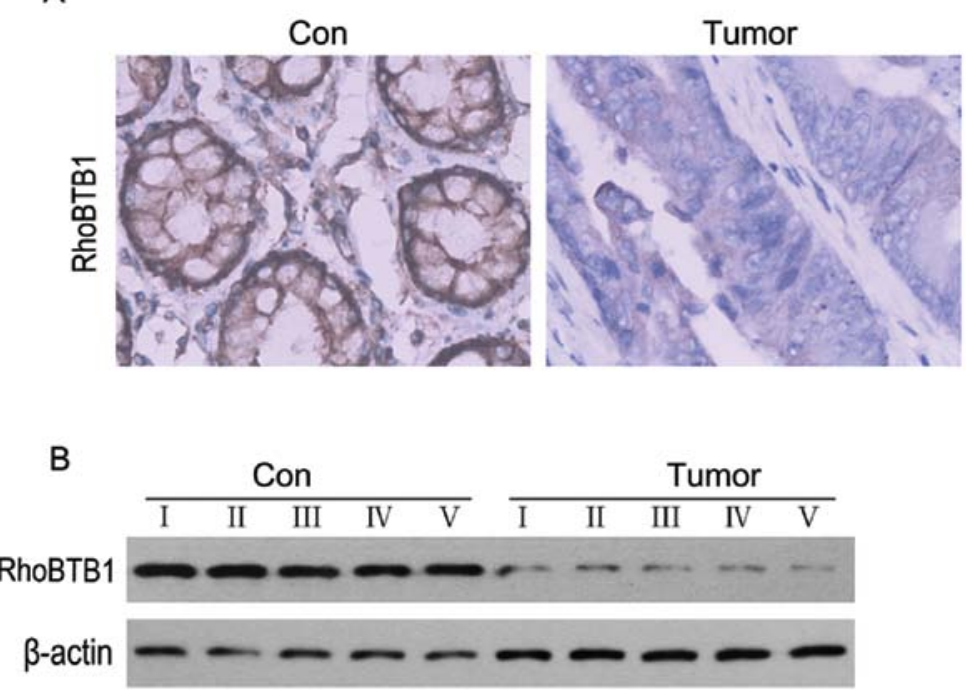

Figure 5. RhoBTB1 was decreased in human colon cancer tissues. (A) A drastic decrease in RhoBTB1 immunoreactivity is noted in colon cancer tissue compared with normal tissue. (B) RhoBTB1 expressed in colon cancer tissues were detected by western blotting.

clearly decreased compared to normal colon tissues. These results suggested that the reduction of RhoBTB1 might attribute to colon tumorigenesis.

Inhibition of RhoBTB1 is responsible for the tumor promoting effects of miR-31. To further confirm that miR-31-induced colon tumor progression is mediated by RhoBTB1, we knockdown RhoBTB1 in HT29 cells by RNAi. As shown in Fig. 6B, RhoBTB1 mRNA was effectively inhibited after RhoBTB1 siRNA transfected, and MTT assay results showed HT29 proliferation was induced with suppression of RhoBTB1. Soft agarose colony forming assay results showed inhibition of RhoBTB1 promoted colon cancer cell clonal growth. These data proved that the miR-31 promoted tumor development at least partly through suppressing tumor supressor RhoBTB1.

\section{Discussion}

miRNAs are endogenously expressed small non-coding RNAs, which control gene expression by targeting messenger
RNAs. It has been estimated that in human, genes code for miRNAs account for approximately $3 \%$, which may regulate $30 \%$ of the protein-coding genes $(33,34)$. Convincing evidence has shown that miRNAs are often dysregulated in human cancers and play pivotal roles in tumorigenesis and progression $(35,36)$.

miR-31 was found to be aberrant in many cancers, including colon cancer (20-26). In this study, we demostrated that miR-31 was significantly upregulated in human colon cancer. As shown in Fig. 1, compared to normal colon tissues, miR-31 was increased in colon cancer tissues and colon cancer cell line HT29 and SW480. ISH assays further confirmed that miR-31 was overexpressed in colon cancer tissues. The results were in consistent with the previous studies. miR-31 has been reported to be associated with metastasis in many tumor types. For example, miR-31 was markedly upregulated in oral squamous cell carcinoma (OSCC) and mediated oral oncogenesis (37), and it has been reported that miR-31 promotes lung tumorigenesis by targeting tumor supressors LATS2 and PPP2R2A (23). To examine effect of miR-31 
A
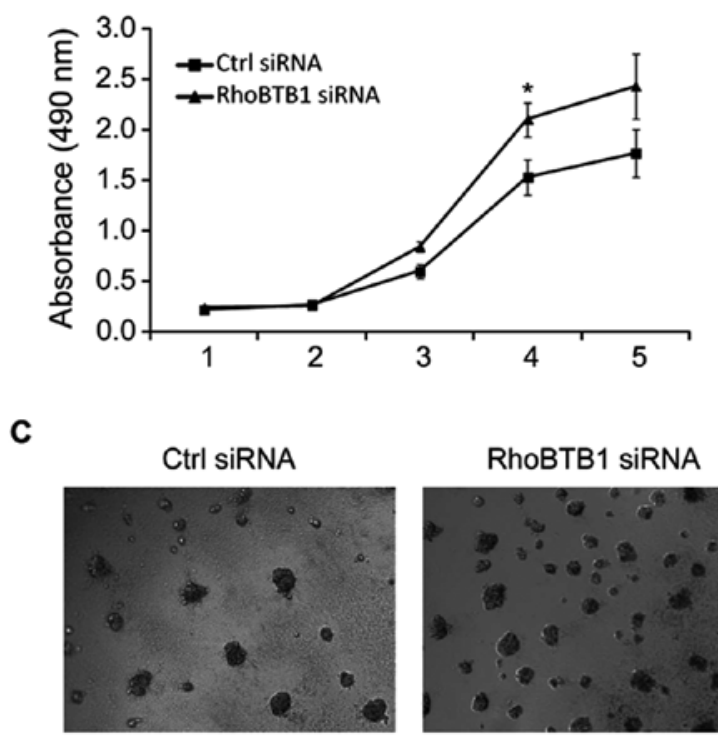

B
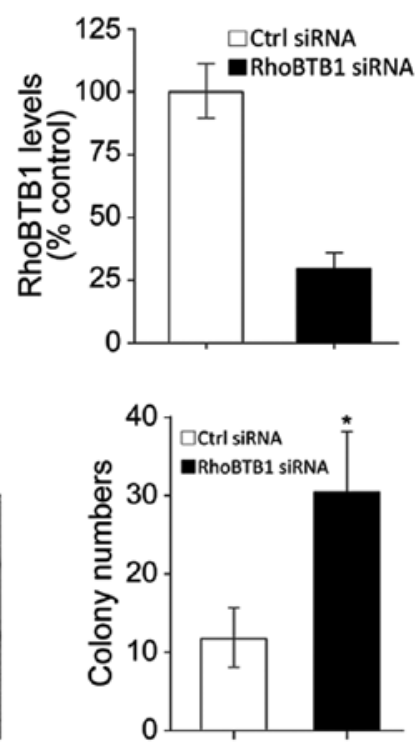

Figure 6. Inhibition of RhoBTB1 is responsible for the tumor promoting effects of miR-31. (A and B) Inhibition of RhoBTB1 by RNAi mimics the function of miR-31. HT29 cells were transfected with RhoBTB1 siRNA, and assayed by MTT. Suppression of RhoBTB1 induces HT29 cell proliferation. The mRNA level of RhoBTB1 is shown (B) by real-time analysis. ${ }^{*} \mathrm{P}<0.05$. (C) Inhibition of RhoBTB1 by RNAi promotes human colon cancer cell clonal growth. Colony formation was suppressed in HT29 cells relative to controls by engineered knockdown of RhoBTB1 via RhoBTB1 siRNA transfection. Representative colonies are displayed. ${ }^{*} \mathrm{P}<0.05$.

depression on colon cancer growth and proliferation, we used LNA anti-miR31 to knockdown miR-31 in HT29 cells. MTT assay results showed HT29 cells proliferation was increased after LAN miR-31 transfection (Fig. 2A). With knockdown of miR-31, HT29 clonal formation ability was inhibited (Fig. 2B). The results above suggested that miR-31 acts primarily as an oncogene in colon cancer. Since miRNAs affect cellular activities by targeting their downstream targets, we next looked for the targets of miR-31.

In order to identify miR-31 target genes, we used TargetScan software, the results showed that tumor supressor RhoBTB1 might be a target of miR-31. RhoTBT1 belongs to RhoBTB subfamily. RhoBTB proteins are atypical members of Rho family of small GTPases. Rho GTPases act as molecular switches in regulating actin filament system, and are considered as major regulators of cytoskeletal remodeling, while increasing evidence points to participation also in the regulation of cell cycle progression and apoptosis, and Rho GTPases were also reported to be involved in tumor development (38-40).

The RhoBTB subfamily was identified during the study of the genes encoding Rho-related proteins in the lower eukaryote Dictyostelium discoideum in 2001 (41), thus it was the most recent addition to the Rho family. Unlike other typical RhoGTPases, RhoBTB proteins do not act as a main regulator in actin filament system. RhoBTB2 and RhoBTB1, have been proposed as tumor supressors. RhoBTB2 was the first to be proposed as a candidate tumor suppressor being identified as the gene homozygously deleted at 8p21 in breast cancer and resulted in cell growth inhibition. RhoBTB1, which is located at the $10 \mathrm{q} 21$ region, was suggested to have a role in head an neck squamous cell carcinomas (HNSCC) development. Levent and coworkers found that in 52 HNSCC,
$42 \%$ showed $\mathrm{LOH}$ at $10 \mathrm{q} 23.1$, and in 5 tumors decreased expression of RhoBTB1 was accompanied by LOH.

Although it has been demostrated that RhoBTB1 was involved in tumorigenesis, scarce knowledge exists on its role in colon cancer, so we examined the level of RhoBTB1 protein expression in colon cancer tissues. ICH results showed that, compared to normal colon tissues, RhoBTB1 was dramatically decreased in colon cancer (Fig. 5). To further examine whether the depressed RhoBTB1 was responsible for the tumor promoting effects of miR-31, RhoBTB1 was silenced by RNAi, as indicated in Fig. 6A, supression of RhoTBT1 in HT29 inhibited cell proliferation, which mimics the function of miR-31. The knockdown of RhoTBT1 also promoted HT29 cells clonal growth.

In conclusion, our study suggests high levels of miR-31 are involved in colon cancer development, and we discovered tumor supressor RhoBTB1 as a new target of miR-31. Overexpression of miR-31 promotes tumor proliferation through depressing the tumor supressor RhoBTB1. These observations shed new light on mechanisms underlying development of colon cancer and supply potential novel therapeutic targets in inhibiting colon tumorigenesis.

\section{Acknowledgements}

This study was supported by grants from Jilin Provincial Sciecce and Technology Department (no. 20070727-01) and the International Partnership.

\section{References}

1. Jemal A, Siegel R, Xu J and Ward E: Cancer statistics, 2010. CA Cancer J Clin 60: 277-300, 2010. 
2. Colorectal Cancer Survival by Stage: National Cancer Intelligence Network, 2009

3. Figer A, Perez-Staub N, Carola E, et al: FOLFOX in patients aged between 76 and 80 years with metastatic colorectal cancer: an exploratory cohort of the OPTIMOX1 study. Cancer 110: 2666-2671, 2007.

4. Becker H: Surgery of colorectal carcinoma. Praxis 84: 1371-1372, 1995.

5. Jemal A, Siegel R, Ward E, Murray T, Xu J, Smigal C and Thun MJ: Cancer statistics, 2006. CA Cancer J Clin 56: 106-130, 2006.

6. Tsai HL, Yeh YS, Yu FJ, Lu CY, Chen CF, Chen CW, Chang YT and Wang JY: Predicting factors of postoperative relapse in T2-4N0M0 colorectal cancer patients via harvesting a minimum of 12 lymph nodes. Int J Colorectal Dis 24: 177-183, 2009.

7. Longo WE and Johnson FE: The preoperative assessment and postoperative surveillance of patients with colon and rectal cancer. Surg Clin North Am 82: 1091-1108, 2002.

8. Baraniskin A, Birkenkamp-Demtroder K, Maghnouj A, et al: MiR-30a-5p suppresses tumor growth in colon carcinoma by targeting DTL. Carcinogenesis 33: 732-739, 2012.

9. Friedman RC, Farh KK, Burge CB and Bartel DP: Most mammalian mRNAs are conserved targets of microRNAs. Genome Res 19 : 92-105, 2009.

10. Reinhart BJ, Slack FJ, Basson M, Pasquinelli AE, Bettinger JC, Rougvie AE, Horvitz HR and Ruvkun G: The 21-nucleotide let-7 RNA regulates developmental timing in Caenorhabditis elegans. Nature 403: 901-906, 2000.

11. Chen CZ, Li L, Lodish HF and Bartel DP: MicroRNAs modulate hematopoietic lineage differentiation. Science 303 83-86, 2004

12. Lai EC: Micro RNAs are complementary to 3'UTR sequence motifs that mediate negative post-transcriptional regulation. Nat Genet 30: 363-364, 2002.

13. Engels BM and Hutvagner G: Principles and effects of microRNAmediated post-transcriptional gene regulation. Oncogene 25 6163-6169, 2006.

14. Cheng AM, Byrom MW, Shelton J and Ford LP: Antisense inhibition of human miRNAs and indications for an involvement of miRNA in cell growth and apoptosis. Nucleic Acids Res 33: 1290-1297, 2005.

15. Calin GA, Ferracin M, Cimmino A, et al: A MicroRNA signature associated with prognosis and progression in chronic lymphocytic leukemia. N Engl J Med 353: 1793-1801, 2005.

16. Bushati $\mathrm{N}$ and Cohen SM: microRNA functions. Annu Rev Cell Dev Biol 23: 175-205, 2007.

17. Garzon R, Calin GA and Croce CM: MicroRNAs in cancer Annu Rev Med 60: 167-179, 2009.

18. Slaby O, Svoboda M, Michalek J and Vyzula R: MicroRNAs in colorectal cancer: translation of molecular biology into clinical application. Mol Cancer 8: 102, 2009.

19. Schetter AJ and Harris CC: Alterations of microRNAs contribute to colon carcinogenesis. Semin Oncol 38: 734-742, 2011.

20. Bandres E, Cubedo E, Agirre X, et al: Identification by real-time PCR of 13 mature microRNAs differentially expressed in colorectal cancer and non-tumoral tissues. Mol Cancer 5: 29, 2006.

21. Slaby O, Svoboda M, Fabian P, Smerdova T, Knoflickova D, Bednarikova M, Nenutil R and Vyzula R: Altered expression of miR-21, miR-31, miR-143 and miR-145 is related to clinicopathologic features of colorectal cancer. Oncology 72: 397-402, 2007.
22. Wong QW, Lung RW, Law PT, Lai PB, Chan KY, To KF and Wong N: MicroRNA-223 is commonly repressed in hepatocellular carcinoma and potentiates expression of Stathmin1. Gastroenterology 135: 257-269, 2008.

23. Liu X, Sempere LF, Ouyang H, et al: MicroRNA-31 functions as an oncogenic microRNA in mouse and human lung cancer cells by repressing specific tumor suppressors. J Clin Invest 120: 1298-1309, 2010

24. Liu X, Chen Z, Yu J, Xia J and Zhou X: MicroRNA profiling and head and neck cancer. Comp Funct Genomics, 837514, 2009.

25. Valastyan S, Reinhardt F, Benaich N, Calogrias D, Szasz AM, Wang ZC, Brock JE, Richardson AL and Weinberg RA: A pleiotropically acting microRNA, miR-31, inhibits breast cancer metastasis. Cell 137: 1032-1046, 2009.

26. Laurila EM, Sandström S, Rantanen LM, Autio R and Kallioniemi A: Both inhibition and enhanced expression of miR-31 lead to reduced migration and invasion of pancreatic cancer cells. Genes Chromosomes Cancer 51: 557-568, 2012.

27. Cottonham CL, Kaneko S and Xu L: miR-21 and miR-31 con-verge on TIAM 1 to regulate migration and invasion of colon carcinoma cells. J Biol Chem 285: 35293-35302, 2010.

28. Wang CJ, Stratmann J, Zhou ZG and Sun XF: Suppression of microRNA-31 increases sensitivity to 5-FU at an early stage, and affects cell migration and invasion in HCT-116 colon cancer cells. BMC Cancer 10: 616, 2010.

29. Hamaguchi M, Meth JL, von Klitzing C, et al: DBC2, a candidate for a tumor suppressor gene involved in breast cancer. Proc Natl Acad Sci USA 99: 13647-13652, 2002.

30. Knowles MA, Aveyard JS, Taylor CF, Harnden P and Bass S: Mutation analysis of the $8 \mathrm{p}$ cand idate tumour suppressor genes DBC2 (RHOBT B2) and LZTS1 in bladder cancer. Cancer Lett 225: 121-130, 2005.

31. Cho YG, Choi BJ, Kim CJ, Song JH, Zhang C, Nam SW, Lee JY and Park WS: Genetic analysis of the DBC2 gene in gastric cancer. Acta Oncol 47: 366-371, 2008

32. Beder LB, Gunduz M, Ouchida M, et al: Identification of a candidate tumor suppressor gene RHOBTB1 located at a novel allelic loss region 10q21 in head and neck cancer. J Cancer Res Clin Oncol 132: 19-27, 2006

33. Hwang HW and Mendel JT: MicroRNAs in cell proliferation, cell death, and tumorigenesis. Br J Cancer 94: 776-780, 2006.

34. Lewis BP, Burge CB and Bartel DP: Conserved seed pairing, often flanked by adenosines, indicates that thousands of human genes are microRNA targets. Cell 120: 15-20, 2005.

35. Cho WC: MicroRNAs in cancer from research to therapy. Biochim Biophys Acta 1805: 209-217, 2010.

36. Cho WC: MicroRNAs: potential biomarkers for cancer diagnosis, prognosis and targets for therapy. Int J Biochem Cell Biol 42: $1273-1281,2010$

37. Liu CJ, Tsai MM, Hung PS, et al: miR-31 ablates expression of the HIF regulatory factor FIH to activate the HIF pathway in head and neck carcinoma. Cancer Res 70: 1635-1644, 2010.

38. Sahai E and Marshall CJ: RHO-GTPases and cancer. Nat Rev Cancer 2: 133-142, 2002.

39. Gómez del Pulgar T, Benitah SA, Valerón PF, Espina C and Lacal JC: Rho GTPase expression in tumourigenesis: evidence for a significant link. Bioessays 27: 602-613, 2005.

40. Ellenbroek SI and Collard JG: RhoGTPases: functions and association with cancer. Clin Exp Metastasis 24: 657-672, 2007.

41. Rivero F, Dislich H, Glockner G and Noegel AA: The Dictyostelium discoideum family of Rho-related proteins. Nucleic Acids Res 29: 1068-1079, 2001. 